\title{
La radio como herramienta pedagógica para fomentar la investigación ${ }^{1}$
}

\section{Radio as a pedagogical tool to promote research}

DOI: http://dx.doi.org/10.17981/cultedusoc.9.3.2018.21

Artículo de investigación. Fecha de recepción: 15/06/2018. Fecha de aceptación: 27/11/2018

\author{
Edilberto Chávez²; \\ Adunia Vanegas; Tomás Saucedo; Dabinson Cadena y Roger Mansbach ${ }^{3}$ \\ IED Nicolás Mejía Mendez (Colombia) \\ edilberto.chavez@yahoo.es
}

Para citar este artículo:

Chávez, E., Vanegas, A., Saucedo, T., Cadena, D. y Mansbach, R. (2018). La radio como herramienta pedagógica para fomentar la investigación. Cultura. Educación y Sociedad 9(3), 179-188. DOI: http://dx.doi.org/10.17981/cultedusoc.9.3.2018.21

\section{Resumen}

La radio es un medio de comunicación que permite conocer e informar a las personas sobre temas de interés, y permite la participación y actualización de conocimiento, resultado una estrategia de innovación en los sectores educativos. El presente estudio entonces tiene como objetivo utilizar la radio como herramienta pedagógica para fomentar la investigación en la comunidad de Urquijo, Municipio de Guamal - Magdalena. Se asume un abordaje cualitativo, desde el paradigma histórico hermenéutico, de alcance descriptivo. Los participantes fueron los estudiantes de la Institución Educativa Nicolás Mejía Méndez. Dentro de los hallazgos encontrados, existe gran afinidad entre los estudiantes y las actividades radiales, lo cual indica que el proyecto tiene un alto nivel de aceptación, sin embargo se debe enfatizar en que la emisora no reemplaza la actividad académica.

Palabras clave: Proyecto pedagógico, radio escolar, investigación; herramienta pedagógica; TIC.

\section{Abstract}

The radio is a means of communication that allows to know and inform people about topics of interest, and allows the participation and updating of knowledge, resulting in an innovation strategy in the educational sectors. The present study then aims to use the radio as a pedagogical tool to promote research in the community of Urquijo, Municipality of Guamal - Magdalena. It assumes a qualitative approach, from the hermeneutical historical paradigm, of descriptive scope. The participants were the students of the Nicolás Mejía Méndez Educational Institution. Among the findings found, there is great affinity between the students and the radio activities, which indicates that the project has a high level of acceptance, however it should be emphasized that the station does not replace the academic activity.

Keywords: Pedagogical project, school radio, research; pedagogical tool; TIC.

\footnotetext{
1 Este artículo ha sido derivado del Programa de Fortalecimiento de la Cultura Ciudadana y Democrática CT+I a través de la IEP apoyada en TIC en el Departamento de Magdalena: CICLON.

2 Líder del grupo de investigación "La radio: educación, diversión y poder".

3 Docentes pertenecientes al grupo de investigación La radio; educación, diversión y poder de la IED Nicolás Mejía Mendez. Ciclón Magdalena. Estrategia $\mathrm{N}^{\circ} 2$.
}

- The author; licensee Universidad de la Costa - CUC 


\section{Introducción}

Una emisora de radio en el colegio puede convertirse en un excelente complemento para la labor educativa, tanto para estudiantes como para profesores, siendo uno de los más idóneos instrumentos para la transformación del espacio, la gestión, las relaciones y las dinámicas conflictivas y convivientes en la escuela, espacio en el que hay que continuar pensando y diferenciando al sujeto joven.

Tener este medio de comunicación en la escuela es muy satisfactorio y un desafío que pone en juego destrezas, fantasía, culturas, compromisos y una gran dosis de locura a la prestación de la comunicación, siendo este un pionero y competente en este campos del dinamismo humano. Desde este importante medio, se pueden fomentar procesos como la investigación, elemento que impacta de manera significativa al currículo de la Institución Educativa Departamental Nicolás Mejía Méndez y por consiguiente a la comunidad del corregimiento de Urquijo, gracias a la estrategia que ha implementado el programa Ciclón que ha ubicado el acto de investigar al alcance de los niños (as) y jóvenes.

La intención no es que el proyecto de la emisora compita con los colosos de los medios de comunicación masivos que se movilizan por intereses comerciales, tal como expresan Valenzuela y Rochin (2013), los medios de comunicación en diversas ocasiones tienen intereses vinculados con grupos de poder, actores políticos y el mismo gobierno, y aun cuando la UNESCO según Nuñez y Ravina (2017), pretende que los países establezcan a nivel internacional un marco ético y reglamentario destinado a la protección jurídica en los medios de comunicación, sobre todo, en las redes sociales, por lo tanto el propósito; es que brinde unos espacios formativos para la comunidad educativa (estudiantes, docentes, la familia y directivas de la institución educativa).
El propósito fundamental es utilizar la emisora escolar para fomentar el desarrollo de un currículo innovador a través de la IEP (Investigación como estrategia pedagógica), con las herramientas de las TIC y formar métodos efusivos, organizativos y didácticos intactos a favor de la entidad pedagógica, que admita renovar los niveles de armonía, solucionar problemas, mejorar las relaciones y optimizar la capacidad de convivenvia de los estudiantes en la institución y todos los que están relacionados con esta comunidad Educativa Nicolás Mejía Méndez.

\section{Herramientas Pedagógicas}

La Real Academia de la lengua Española define el término pedagogía de la siguiente manera: Ciencia que se ocupa de la Educación y de la Enseñanza. En general, lo que enseña y educa por doctrina o ejemplos.

De acuerdo con Cortés, Montoya y Chávez (2008), el significado etimológico de Pedagogía está orientado a enseñar. La Pedagogía es hoy en día un término mundialmente conocido, ya que las nuevas generaciones y la aplicación de nuevas tecnologías han conducido al ser humano a una constante carrera por aprender más.

La Pedagogía es una ciencia muy compleja y que debe ser vista con cuidado, ya que la pedagogía estudia todo lo relacionado con la educación y la posición de los valores de conocimiento en el ser humano. Derivada de los griegos "Paidon" que significa "Niño" y "Gogos" que quiere decir "Conducir" la palabra Pedagogía nos hace referencia a su visión, evocada netamente a la formación de métodos que garanticen una educación de alto valor. Cuando se habla de Pedagogía en una institución se está haciendo un estudio analítico de los procesos de enseñanza que existen, para reforzar o elaborar mejores estrategias que el ayudaran al niño estudiante a absorber la máxima cantidad de conocimiento posible. 
Cortés, Montoya y Chávez (2008), en relación a la importancia de implementar herramientas pedagógicas en el proceso enseñanza - aprendizaje, comentan que dicho proceso debe ser riguroso y actualizarse con periocidad, El material debe ser lo más autosuficiente posible, en el sentido de que el alumnado no necesite recurrir a ningún otro material, con el objetivo de facilitar su utilización, los objetivos que se pretenden, empleando para ello la claridad y precisión de lo que se espera u pretende de acuerdo a la actividad dispuesta y el conocimiento previo delos estudiantes.

No obstante hay otras herramientas con las cuales los docentes aplican estrategias para las actividades deben ser comprensibles, variadas, viables y significativas para la comprensión de los temas, Para esto se desarrollan herramientas pedagógicas que los niños usan en las aulas y espacios comunes de los centros educativos. Estas herramientas constituyen instrumentos pedagógicos que los maestros elaboran con el fin de acompañar el aprendizaje de los niños y así potenciar su desarrollo. Y que son potenciales herramientas pedagógicas para transformar la realidad. Por tal razón los autores buscan poner de manifiesto y fortalecer el sistema educativo, para que se utilicen más y mejores estrategias que apoyen los procesos de Enseñanza Aprendizaje dentro y fuera del aula de clase.

La Radio como herramienta pedagógica para Mata, (1988) citado en Padilla (2015), describe los conceptos que predominan en cuanto a la radio, entendida como medio de difusión, práctica significante y como experiencia cultural (p. 15.) En cuanto al primer concepto predominante, la radio ha sido entendida como medio de difusión, partiendo del modelo comunicacional de Shannon y Weaver, los modelos psicológicos y sociológicos de la acción de corte conductista y los modelos lingüísticos y semióticos positivistas Mata caracteriza la radio como un canal tecnológico, un conjunto de men- sajes y una institución social sujeta a leyes, regulaciones, normas y cambios históricos.

Por otra parte partiendo de los aportes de la semiótica textual y de diferentes versiones de la teoría del discurso, aparece otro concepto predominante que caracteriza la radio como espacio de compleja interacción entre emisores y receptores, entre medios y públicos. Por lo tanto como espacio clave para la constitución de identidades individuales y colectivas a través del juego de múltiples interpretaciones, es decir como práctica significante. Finalmente esta autora basada en los aportes de la teoría de la cultura, particularmente de Raymond Williams y los 'Cultural Studies', caracteriza los medios masivos entre ellos la radiocomo hechos o experiencias culturales que tienen una dimensión técnica, institucional, política y específicamente significante $o$, si se quiere, comunicativa.

De acuerdo con Padilla (2015), la pertinencia académica de la radio escolar como estrategia didáctica, parte de la intencionalidad de potenciar las competencias comunicativas de los estudiantes, inicialmente las competencias básicas: escuchar y hablar. Contrario a lo que podría pensarse en el ejercicio de la producción radial no se quedan de lado las competencias de leer y escribir, pero más allá de estas competencias básicas hay otras competencias que potencia la radio, las cuales han sido enunciadas y caracterizadas por diferentes teóricos y pedagogos; la radio es educativa en la medida que se utiliza para motivar a sus estudiantes, presentado como un recurso distinto a los medios tradicionales, así mismo hacer frente a las carencias que el alumnado presenta en cuanto al conocimiento de la lengua oral y como lengua escrita. Al tiempo la radio educativa permite que se sumen todos los sectores de la comunidad escolar, el centro de familias, el alumnado, creando así un proyecto comunicativo radial. De esa manera los estudiantes ven ante sí una oportunidad de 
poder participar en programas de radio que escucharan sus compañeros y sus padres. (Castilla, Gómez, \& Paniagua, 2007, p. 1).

Otros teóricos de la comunicación y la educación, han resaltado los diferentes aportes de la formación auditiva a la capacitación del individuo. En su artículo "Educar a través de la radio", (Rodero, 2008, p. 1) resalta el lenguaje auditivo como estimulante de la imaginación y asegura que la formación auditiva potencia el procesamiento analítico, desarrolla la capacidad discursiva, fomenta la capacidad de escucha y permite la interacción con el emisor.

Partiendo de estos aportes de la formación auditiva a la capacitación del individuo, Rodero valora la radio como fuente estimuladora de la imaginación, ya que al no tener el componente visual posibilita la creación de imágenes que estimula la imaginación. Agrega la autora que la radio es el medio que mejor fomenta la capacidad de escucha: la radio sirve para educar, informar, formar y enseñar está dada por sus características; por sus ventajas y desventajas como medio de comunicación masiva. (Rodero, 2008. p. 107). Finalmente la autora resalta la radio como escenario propicio para la capacitación de la expresión oral.

El Ministerio de Educación Nacional (2017), en su espacio informativo "Al Tablero", en el artículo "Uso pedagógico de tecnologías y medios de comunicación. Exigencia constante para docentes y estudiantes", señala que la relevancia de que los creadores del lenguaje radiofónico son principalmente se controlan las variables intervinientes. El Ministerio avanza el proyecto de implementar los medios de comunicación de la mano de los maestros. Es entonces que la radio escolar puede llegar a convertirse en herramienta que propicie el desarrollo de competencias investigativas, comunicativas eficaces, entre otras que permitan convertirse en alternativas de resolución de conflicto y promotoras de la convivencia pacífica.
A nivel intelectual, la radio establece actividades formativas que integran técnicas radiofónicas ayudan a comprender el entorno, posibilitan el aprendizaje en todos los niveles educativos y permiten generar inquietudes creativas en los participantes. La comunicación sirve para educar, informar, formar y enseñar está dada por sus características; por sus ventajas y desventajas como medio de comunicación masiva. La radio como medio de comunicación abre el horizonte conceptual, en lo político, lo social, lo cultural, lo económico. Promueve una formación, se aprende a manejar discurso, y propende a la comunicación asertiva desde el hablante y el oyente.

De acuerdo con González (2010), la radio escolar, "es una fusión, de elementos de la radio comunitaria y de la radio educativa, con el ingrediente adicional y especial, de que es producida por niños y jóvenes que se encuentran dentro de una formación académica. En esta etapa de la formación, es cuando se crean criterios personales, se establecen las formas de socialización y de interacción con el mundo que los rodea" p.p11.

Sevillano (2012) señala que esta dimensión ha sido tratada por Bartolomé (1998), Sevillano (Coord. 2009), Cebrián (2007 y 2008) entre otros. Se puede afirmar que la radio educativa es una modalidad radiofónica desde la cual se intenta alcanzar objetivos no comerciales y se orienta especial y directamente hacia una finalidad de carácter social.

\section{Metodología}

La investigación desarrollada es de tipo cualitativa, de alcance descriptivo, soportada desde el paradigma socio crítico, pues tal como lo expresa Cerda (2003) la idea es interpretar y comprender os fenómenos estudiados. El diseño de la investigación es Transeccional porque se recolectan datos en un solo momento, en un tiempo único. 


\section{Escenarios y actores}

Los participantes del estudio fueron los estudiantes de la Institución Nicolás Mejía Mendez con una muestra inicial de 20 participantes.

\section{Técnicas e instrumentos de recolección de la información}

Las técnicas específicas en la recolección de información, fueron la observación registrada en un diario de campo y la entrevista estructurada.

\section{Procedimiento}

El diseño de la Entrevista estructurada se formuló de manera que se obtuviera información precisa sobre el objeto de investigación, develando información respecto al parecer de los sujetos participantes en virtud del proyecto pedagógico de radio educativa.

El diseño de las preguntas, tuvo una guía que permitiera elaboración definitiva del cuestionario y del plan operativo de la entrevista basándose en propósitos específicos de la investigación, se realizó una validación presentada a expertos y fue aplicada a estudiantes de diferentes grados de la I. E. D. R. Nicolás Mejía Méndez.

La entrevista se llevó a cabo de acuerdo a un proceso que contó con la participación de los docentes investigadores, lluvia de ideas, la redacción y la selección de preguntas, hasta la validación de la misma.

\section{Resultados}

A continuación se presentan los hallazgos derivados de la aplicación del instrumento de recolección de la información (entrevista estructurada y observación). Los hallazgos fueron posibles a través de la siguiente pregunta orientadora. ¿De qué manera puede ser utilizada la radio para fomentar la investigación en la comunidad de Urquijo, Guamal - Magdalena?

El instrumento fue aplicado a 20 estudiantes de diferentes grados de la I. E. D. R. Nicolás Mejía Méndez.

\begin{tabular}{|c|c|c|c|}
\hline $\begin{array}{l}\text { Categoría de } \\
\text { estudio }\end{array}$ & $\begin{array}{l}\text { Pregunta } \\
\text { orientadora }\end{array}$ & $\begin{array}{l}\text { Discurso de los } \\
\text { actores }\end{array}$ & Articulación y sistematización teórica \\
\hline La radio & $\begin{array}{l}\text { ¿Qué opinión } \\
\text { tienes de } \\
\text { la Emisora } \\
95.5 \text { La Voz } \\
\text { Estudiantil? }\end{array}$ & $\begin{array}{l}\text { Me parece } \\
\text { buena.".Nos ayuda } \\
\text { a aprender a hablar } \\
\text { por micrófono", "Me } \\
\text { gusta cuando los } \\
\text { profesores nos llevan } \\
\text { a la Emisora", "Que } \\
\text { es buena cuando } \\
\text { nos ayudan a hacer } \\
\text { las tareas", "Que } \\
\text { la Emisora del } \\
\text { colegio pone a la } \\
\text { gente alegre por las } \\
\text { mañanas", }\end{array}$ & $\begin{array}{l}\text { De acuerdo a los hallazgos encontrados en la } \\
\text { entrevista aplicada a los estudiantes, se puede } \\
\text { inferir que les gusta la Emisora porque le } \\
\text { encuentran alguna utilidad. } \\
\text { De acuerdo con González (2010), el ingrediente } \\
\text { adicional y especial de la Radio Educativa es } \\
\text { que es co - producida por niños y jóvenes que se } \\
\text { encuentran dentro de una formación académica. } \\
\text { En esta etapa de la formación, es cuando se } \\
\text { crean criterios personales, se establecen las } \\
\text { formas de socialización y de interacción con el } \\
\text { mundo que los rodea. } \\
\text { Lo anterior, explica la necesidad de } \\
\text { implementar programas y actividades como } \\
\text { la Radio Escolar, pero más importante aún es } \\
\text { permitir que los estudiantes sean asertivos } \\
\text { y críticos frente a lo que se está realizando } \\
\text { en la institución, porque de esta manera se } \\
\text { corrigen los errores y se avanza hacia la calidad } \\
\text { académica y personal en la institución. }\end{array}$ \\
\hline
\end{tabular}




\begin{tabular}{|c|c|c|c|}
\hline La radio & $\begin{array}{l}\text { ¿Qué tipo de } \\
\text { programas te } \\
\text { gustaría que } \\
\text { transmitieran } \\
\text { en la Emisora } \\
95.5 \text { Voz } \\
\text { Estudiantil? }\end{array}$ & $\begin{array}{l}\text { "Programas } \\
\text { educativos", "Que } \\
\text { tengan cultura", } \\
\text { "Programas que } \\
\text { enseñen algo } \\
\text { bueno, que dejen un } \\
\text { mensaje., "Que nos } \\
\text { enseñen ortografía", } \\
\text { "Que tengan que ver } \\
\text { con las capacidades } \\
\text { artísticas de los } \\
\text { compañeros", } \\
\text { "Programas de } \\
\text { concursos de } \\
\text { matemáticas } \\
\text { y Lenguaje", } \\
\text { "Programas que } \\
\text { muestren nuestro } \\
\text { folklor de nuestra } \\
\text { población". }\end{array}$ & $\begin{array}{l}\text { Lo expresado por los Estudiantes, conlleva } \\
\text { a inferir que las preferencias en relación a } \\
\text { los contenidos que desearían escuchar en la } \\
\text { Emisora están relacionadas con la Educación, } \\
\text { la Cultura, las situaciones de la sociedad local, } \\
\text { los deportes, y la entretención, dejando ver el } \\
\text { interés que demuestran porque el contenido } \\
\text { radiofónico sea formativo. } \\
\text { Al respecto, Sevillano (2012), afirma que la } \\
\text { radio educativa, en cualquiera de sus formas, } \\
\text { ha de orientarse precisamente hacia un } \\
\text { objetivo de carácter social, y resalta el rol que } \\
\text { debe desempeñar una Emisora Escolar, como } \\
\text { espacio donde se construyen las identidades } \\
\text { culturales a partir de contenidos relacionados } \\
\text { con la actividad local, pensando siempre en la } \\
\text { proyección e impacto que produzca lo que se } \\
\text { Emite al Exterior. }\end{array}$ \\
\hline Investigación & $\begin{array}{l}\text { ¿Alguna } \\
\text { vez has } \\
\text { participado en } \\
\text { proyectos de } \\
\text { investigación? }\end{array}$ & $\begin{array}{l}\text { "Si“, "Desde que } \\
\text { está Ciclón estoy } \\
\text { participando en } \\
\text { un proyecto con } \\
\text { la Profe., ".Si he } \\
\text { participado, porque } \\
\text { ahora en casi todas } \\
\text { las clases se habla } \\
\text { del proyecto". }\end{array}$ & $\begin{array}{l}\text { En relación a las anteriores respuestas, la } \\
\text { autora Ciro (2012), resalta las bondades de un } \\
\text { enfoque de enseñanza basado en proyectos, } \\
\text { afirmando que se construye sobre las fortalezas } \\
\text { individuales de los estudiantes y les permite } \\
\text { explorar sus áreas de interés dentro del marco } \\
\text { de un currículo establecido, además constituye } \\
\text { un modelo de instrucción auténtico en el que los } \\
\text { estudiantes planean, implementan y evalúan } \\
\text { proyectos que tienen aplicación en el mundo real } \\
\text { más allá del aula de clase. }\end{array}$ \\
\hline Investigación & $\begin{array}{l}\text { ¿Te gustaría } \\
\text { participar, } \\
\text { o seguir } \\
\text { participando } \\
\text { en } \\
\text { proyectos de } \\
\text { investigación? }\end{array}$ & 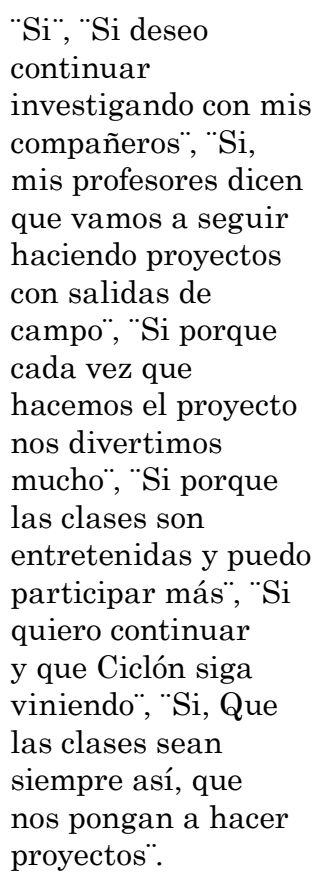 & $\begin{array}{l}\text { Al respecto, Ciro (2012), señala que la } \\
\text { propuesta del trabajo por proyectos de } \\
\text { investigación, pretende brindar un apoyo en el } \\
\text { ámbito educativo, para la implementación de } \\
\text { una metodología que complemente los temas } \\
\text { de las clases teóricas con la aplicación de } \\
\text { proyectos diseñados para este fin y a la vez, } \\
\text { incentivar mediante su realización, actividades } \\
\text { como: investigación, planeación, búsqueda de } \\
\text { soluciones, trabajo cooperativo y actitudes como: } \\
\text { autorregulación, disciplina y perseverancia, } \\
\text { entre otros elementos que benefician la } \\
\text { formación integral de los estudiantes. } \\
\text { En tal sentido, la autora enfatiza en que } \\
\text { trabajar por proyectos no excluye la importancia } \\
\text { de la teoría, es decir, no significa perder el } \\
\text { rigor de la enseñanza, ya que los contenidos } \\
\text { siguen siendo la base del aprendizaje, solo que } \\
\text { el secreto está en hallar el punto de equilibrio } \\
\text { entre teoría y práctica. }\end{array}$ \\
\hline
\end{tabular}




\begin{tabular}{|c|c|c|c|}
\hline $\begin{array}{l}\text { La radio } \\
\text { investigación }\end{array}$ & $\begin{array}{l}\text { ¿Te gustaría } \\
\text { que en la } \\
\text { Emisora } \\
95.5 \text { La voz } \\
\text { Estudiantil } \\
\text { transmitieran }\end{array}$ & $\begin{array}{l}\text { "Si me gustaría } \\
\text { porque la gente } \\
\text { conoce lo que } \\
\text { estamos haciendo., } \\
\text { "Si porque la } \\
\text { Emisora nos motiva } \\
\text { para presentar } \\
\text { los trabajos bien", } \\
\text { "Me gustaria que } \\
\text { todas las personas } \\
\text { supieran los } \\
\text { proyectos que hago", } \\
\text { "Si, yo quiero que } \\
\text { todas las clases } \\
\text { sean en la Emisora", } \\
\text { "Si porque es muy } \\
\text { importante que } \\
\text { le mostremos a } \\
\text { la gente que nos } \\
\text { preocupamos por el } \\
\text { medio ambiente.. }\end{array}$ & $\begin{array}{l}\text { Al respecto Villamizar (2002), destaca que } \\
\text { la experiencia de la Radio Escolar no separa } \\
\text { al estudiante de su trabajo formal exigido } \\
\text { por los programas oficiales, antes bien, el } \\
\text { cumplimiento de tales exigencias programáticas } \\
\text { se convierte en el principal proveedor de temas, } \\
\text { problemas y búsquedas para la realización de } \\
\text { los "programas". Reiteremos que el trabajo de } \\
\text { la "radio escolar" no es en ningún caso una } \\
\text { "actividad complementaria", sino una opción } \\
\text { distinta para el abordaje de la labor cotidiana } \\
\text { del aula. } \\
\text { Es imprescindible trastocar la idea del aula } \\
\text { como único espacio para el aprendizaje y abrir a } \\
\text { nuevos escenarios y procesos. } \\
\text { Se requiere rehacer la noción del tiempo para } \\
\text { aprender, de tal suerte que se pueda superar el } \\
\text { lapso cerrado de la clase como exclusivo tiempo } \\
\text { hábil para tal propósito. } \\
\text { Lo que expresa el autor en las anteriores líneas, } \\
\text { es el cuidado que debe tenerse de no caer en } \\
\text { el facilismo de canalizar todas las actividades } \\
\text { a través de la Emisora Escolar, sino que se } \\
\text { convierta esta herramienta en un apoyo, } \\
\text { como los demás recursos tecnológicos, que en } \\
\text { ningún momento pueden convertirse en el fin, } \\
\text { sino en medios que apoyen el desarrollo de las } \\
\text { temáticas académicas como lo determinan los } \\
\text { estándares del Ministerio de Educación. }\end{array}$ \\
\hline TIC & $\begin{array}{l}\text { ¿Cómo } \\
\text { utilizarías } \\
\text { las TIC } \\
\text { (Computador, } \\
\text { celular, } \\
\text { Internet, } \\
\text { Tablet) en la } \\
\text { Emisora del } \\
\text { Colegio? }\end{array}$ & $\begin{array}{l}\text { Para ir consultando } \\
\text { por internet } \\
\text { mientras se } \\
\text { transmiten las } \\
\text { noticias." "Abriendo } \\
\text { los proyectos en } \\
\text { el computador } \\
\text { para leerlos en la } \\
\text { Emisora", "Con } \\
\text { el Chat se puede } \\
\text { conversar también } \\
\text { con los que están } \\
\text { oyendo"., "Se podría } \\
\text { hacer un blog en } \\
\text { el que opinaran } \\
\text { de lo que se está } \\
\text { hablando"." Las } \\
\text { personas podrian } \\
\text { elegir el proyecto } \\
\text { que más les gusta } \\
\text { llamando por } \\
\text { celular". }\end{array}$ & $\begin{array}{l}\text { De acuerdo con Hermosa, (2015), es } \\
\text { incuestionable que los jóvenes de hoy viven } \\
\text { rodeados de ordenadores, tablets, telefonía } \\
\text { móvil, videojuegos, etc. tecnologías que son } \\
\text { consideradas imprescindibles en su día a día. } \\
\text { Esto hace que piensen y procesen la información } \\
\text { de forma distinta a sus antecesores, pues éstos } \\
\text { tienen la percepción de que el contenido que } \\
\text { están dando podrían localizarlo en la red o en } \\
\text { otras fuentes. } \\
\text { El autor expresa que en los ambientes } \\
\text { educativos de la 'Era de la Tecnología“, es } \\
\text { contradictorio tratar de alejar a los estudiantes } \\
\text { de estas herramientas, más bien, se trata } \\
\text { de aprovechar su interés por las prácticas } \\
\text { interactivas y sacar el mejor provecho de } \\
\text { ello. El uso de TIC en la escuela responde a } \\
\text { una demanda socioeducativa generada por el } \\
\text { entorno virtual en el que se encuentra inmerso } \\
\text { el alumnado por pertenecer a la generación en } \\
\text { la que se consolida la cultura de la Tecnología. }\end{array}$ \\
\hline
\end{tabular}

Fuente: elaboración propia. 


\section{Conclusiones}

Los hallazgos descritos corresponden al estudio denominado: "La Radio como herramienta pedagógica para fomentar la investigación en la Comunidad de Urquijo, Municipio de Guamal - Magdalena“.

El propósito general es Utilizar la Radio como Herramienta Pedagógica para Fomentar La Investigación en la Comunidad de Urquijo, Municipio de Guamal - Magdalena, por tanto, se hace necesario realizar una síntesis con base en los propósitos específicos.

Propósito 1: Concientizar a los estudiantes acerca de la importancia de la Emisora Escolar de la Institución para difundir sus proyectos de Investigación. Los estudiantes y docentes reconocen la importancia que tiene la Emisora como medio estratégico para difundir los proyectos de investigación que se realicen en la institución educativa Nicolás Mejía Méndez, mostrando apropiación de conceptos, fundamentos y descubrimiento de técnicas en el uso que debe dársele al medio de comunicación.

En la fase inicial, se hizo necesario el acompañamiento al grupo de investigadores, ya que los docentes tuvieron la necesidad de realizar jornadas de revisión bibliográfica, para conseguir una mayor fundamentación en torno a la temática.

Propósito 2: Proyectar la Emisora Escolar como un instrumento educativo, formativo y receptivo para la comunidad.

En relación al modo como debe ser direccionada la Emisora, sus contenidos y la programación que debe emitirse se tuvo en cuenta el testimonio de los estudiantes, que son los llamados a dinamizar el proceso pedagógico didáctico desde sus investigaciones.

Los procedimientos realizados tuvieron como epicentro la decisión de explotar adecuadamente el importante medio de comunicación con que cuenta la institución, tema que viene siendo tratado por los directivos y docentes que vislumbran la incorporación de la actividad radial al currículo, atendiendo el clamor de los Estudiantes, en comunión con la metodología propuesta desde el programa Ciclón que fomenta el desarrollo de competencias investigativas en los niños, niñas y jóvenes, enfatizando en la necesidad de divulgar el conocimiento resultante a la comunidad.

Propósito 3: Promover el uso de las TIC en la Emisora 95.5 La Voz Estudiantil.

Los Estudiantes mostraron interés desde el inicio del proceso por integrar las TIC y las Redes Sociales a la actividad de la Emisora y fueron sus expresiones las que de alguna manera motivaron a los Docentes a acceder a este adelanto que posicionaría a $\mathbf{9 5 . 5}$ La Voz Estudiantil como pionera en la región.

En algunos momentos existió incredulidad por parte de algunos docentes por considerar que el emprendimiento de dicha labor supone una inversión muy alta, y por otro lado, demandaría preparación y experticia en las personas encargadas, hecho que algunos estudiantes líderes minimizaron, sintiéndose dispuestos a asumir el reto.

Lo anterior, hace alusión a la gran afinidad que existe entre las personas de la nueva generación y la Tecnología, quienes ven con optimismo la realización de proyectos como el que se presenta a través de estas líneas. 


\section{Referencias}

Avendaño, I; Cortés O, Guerrero H. (2015) Competencias sociales y tecnologías de la información y la comunicación como factores asociados al desempeño en estudiantes de básica primaria con experiencia de desplazamiento forzado Diversitas: Perspectivas en Psicología, 11(1). 13-36. Recuperado de http://www.scielo. org.co/pdf/dpp/v11n1/v11n1a02.pdf.

Barrios, A. (2013). La radio en la era digital. Recuperado de: http://www. utadeo.edu.co/files/collections/documents/field_attached_file/andres_ barrios.pdf

Cortés, J. Montoya, N. y Chaves, J. (2008). Herramientas pedagógicas aplicadas en la enseñanza de las figuras de lissajous. Scientia et Technica Año, XIV, (40).

Escobar, N. y Areiza, F. (2009). Proyecto de lectoescritura y emisora La puerta a la formación integral. Institución educativa sol de oriente. Medellín. Colombia. Recuperado de https:/www.medellin.gov.co/irj/go/ $\mathrm{km} /$ docs/educacionNuevo01dic/.../ lectoes.doc

Fernández, S. (2016). Evidencias de fobia, miedo o rechazo hacia la Matemática en estudiantes de décimo año del Colegio El Carmen de Alajuela: Universidad Estatal a Distancia. Recuperado de https://www.uned. ac.cr/ecen/matematica/images/Archivos_Licenciatura/Tesis_y_proy e c t o s / 004 _ S i l e n n e _ Fern\%C3\%A1ndez.pdf .

González, A. (2010). Emisora nuevo horizonte. Experiencia de construcción de una radio escolar. [Tesis]. Pontificia universidad javeriana facultad de comunicación y lenguaje, comunicación social. Bogotá, Colombia.
Hermosa, P. (2015). Influencia de las tecnologías de información y comunicación (TIC) en el proceso enseñanza-aprendizaje: una mejora de las competencias digitales. Revista Científica General José María Córdova, 13(16). 121-132.

Herrera, M. (2014). El "gen egoísta” y la "desesperanza aprendida". Revista Unimar. 34(1).

López, G. (2012). Pensamiento crítico en el aula. Universidad Autónoma del Estado de Morelos. Revista Docencia e Investigación, XXXVII (22). 41-60. Recuperado de http://educacion.to.uclm.es/pdf/revistaDI/3_22_2012.pdf

López, M. (2012). Radio Escolar: El Trocadero: Una Experiencia Educativa, Nuestra Experiencia. Revista Educación y Comunicación, 5. 25 38. C

Núñez, E. y Ravina, R. (2017). Análisis del nivel de competitividad empresarial en el panorama publicitario colombiano fundamentada en el éxito creativo y la responsabilidad ética y jurídica. JURÍDICAS CUC, 13(1). 9-28. http://dx.doi.org/10.17981/juridcuc.13.1.2017.1

República de Colombia. Ministerio de Educación Nacional. (2017). Colombia, la mejor educada en el 2025. Recuperado de: https://www.mineducacion.gov.co/1621/arti cles-355154_foto_portada.pdf

Sevillano, M. (2012). La Radio aliada de la Educación. Revista Educación y Comunicación, 5, 13 - 28. .

Valenzuela, G. - Rochin, L. (2013). Libertad de expresión y su impacto en la democracia. Jurídicas CUC, 9(1), 89-100. 
Vargas, I. (2012). La entrevista en la investigación cualitativa: nuevas tendencias y retos. Centro de Investigación y Docencia en Educación Universidad Nacional, Costa Rica. Recuperado de http://biblioteca.icap.ac.cr/blivi/coleccion_unpan/ bol_diciembre_2013_69/uned/2012/investigacion_cualitativa.pdf
Villamizar, G. (2002). De la escuela en la radio a la radio en la escuela. Sapiens. Revista Universitaria de Investigación, 3(1). 